\title{
Clinical Study Short-Term Efficacy of Ultramicronized Palmitoylethanolamide in Peripheral Neuropathic Pain
}

\author{
Dario Cocito, Erdita Peci, Palma Ciaramitaro, Aristide Merola, and Leonardo Lopiano \\ Department of Neuroscience, AOU Città della Salute e della Scienza di Torino, University of Turin, \\ Via Cherasco 15, 10124 Torino, Italy \\ Correspondence should be addressed to Dario Cocito; dariococito101@gmail.com
}

Received 10 March 2014; Accepted 16 April 2014; Published 20 May 2014

Academic Editor: Donald A. Simone

Copyright (C) 2014 Dario Cocito et al. This is an open access article distributed under the Creative Commons Attribution License, which permits unrestricted use, distribution, and reproduction in any medium, provided the original work is properly cited.

Introduction. This study evaluates the efficacy of palmitoylethanolamide ultramicronized (PEA-um) as an add-on treatment in patients with diabetic or traumatic neuropathic pain (NP). Methods. 30 patients with chronic NP were assessed with Visual Analogue Scale (VAS), NP Symptom Inventory (NPSI), and Health Questionnaire Five Dimensions (EQ-5D), both at baseline and after 10 and 40 days of treatment with $1200 \mathrm{mg} /$ die of PEA-um. All other therapies were maintained stable during the follow-up period. Results. VAS mean score significantly improved within the first 10 days, ranging from $8.20 \pm 1.53$ to $6.40 \pm 1.83(P<0.002)$, with a further decrease to $5.80 \pm 2.04(P<0.001)$ after 40 days of PEA-um administration. Moreover, NPSI total score improved from $5.2 \pm 1.5$ to $3.8 \pm 2.1(P: 0.025)$ and EQ-5D ranged from $-0.30 \pm 0.65$ to $0.5 \pm 0.34(P<0.001)$ between T0 and T2. Conclusions. This study reports the prospective short-term efficacy data of oral PEA-um in patients with diabetic or traumatic NP. A significant improvement was observed both in VAS and NPSI scores and in quality of life scales after 40 days of treatment, although some limitations should be considered, including the short followup and the open-label study design.

\section{Introduction}

Neuropathic pain (NP) due to nerve injury or neuronal dysfunction could be associated with inflammatory reactions and mobilization of the immune system cells [1]. Microglia are thought to coordinate the inflammatory responses of the central nervous system (CNS), while mast cells have a pivotal role in the inflammatory responses of the peripheral nervous system (PNS). Palmitoylethanolamide (PEA-um) is an endogenous fatty acid, which can inhibit the release of proinflammatory mediators from activated mast cells, reducing the recruitment and activation of mast cells at sites of nerve injury [2]. PEA-um demonstrated a significant efficacy on pain in the murine model of diabetic neuropathy [3]. However, few data are currently available in human subjects; sporadic cases have been reported in the literature [4-8], suggesting that PEA-um oral administration may lead to an improvement of NP, reducing the allodynia and hyperalgesia.

In this context our aim was to evaluate the clinical efficacy of oral PEA-um treatment in a cohort of patients with diabetic or traumatic chronic NP, not adequately controlled by other oral conventional therapies. Subjects were included in an open label study, evaluating the efficacy of oral PEA-um administration, as an add-on therapy, after 10 and 40 days of therapy.

\section{Materials and Methods}

A cohort of 30 patients with diabetic or traumatic chronic $\mathrm{NP}$, not controlled by other oral conventional therapies, was included in this study. All subjects suffered from a chronic pain for at least 100 days at the time of recruitment, with a Visual Analogue Scale (VAS) pain score higher than 6 [9], in spite of the best therapeutic regimen with Pregabalin, Gabapentin, and/or Tramadol. All patients included in the study were diagnosed as having an NP, according to the DN4 score $\geq 4$, with an average score of 6.60 (range 4-9), and a stable intensity of pain in the preceding 100 days.

After a preliminary screening, patients were asked to participate at the study and then were evaluated by means of VAS scale, NP Symptom Inventory (NPSI) [10], and health questionnaire five dimensions (EQ-5D) for quality of life [11]. 
TABLE 1: Clinical and demographic characteristics of patients at baseline.

\begin{tabular}{lcc}
\hline & Diabetic neuropathy & Traumatic neuropathy \\
\hline Number of subjects & 23 & 7 \\
Gender & 12 males & 4 males \\
& 11 females & 3 females \\
Age (years old) & $63.87 \pm 13.4$ & $57.14 \pm 7.5$ \\
VAS score & $7.98 \pm 1.7$ & $8.57 \pm 1.4$ \\
NPSI score & $5.4 \pm 1.6$ & $4.9 \pm 2.1$ \\
EQ-5D score & $-0.12 \pm 0.35$ & $-0.78 \pm 1.03$ \\
\hline
\end{tabular}

Main clinical and demographic characteristics of patients affected by diabetic neuropathy and traumatic neuropathy at baseline. VAS: Visual Analogue Scale; NPSI: Neuropathic Pain Symptom Inventory; EQ-5D: health questionnaire five dimensions.

NPSI single items were also evaluated separately: burning (superficial) spontaneous pain (Factor 1); pressing (deep) spontaneous pain (Factor 2); paroxysmal pain (Factor 3); evoked pain (Factor 4); paresthesia/dysesthesia (Factor 5). This study was authorized by the scientific board of our institute and all subjects signed a written informed consent.

Oral PEA-um treatment was initiated at the doses of $1200 \mathrm{mg} / \mathrm{die}$ in sachet formulation for the first 10 days and $1200 \mathrm{mg} /$ die in tablet formulation between the 10th and 40th days. The dosages of all other therapies were maintained stable during the entire duration of the study.

After the first evaluation, performed at the time of inclusion (T0), patients were reassessed at fixed intervals: VAS evaluation was performed in all subjects after 10 days of treatment (T1), while VAS, NPSI, EQ-5D, and DN4 were repeated after 40 days of treatment (T2).

The statistical comparisons at different time points (T0, T1, T2) were performed by means of Friedmann rank sum test and where appropriate using Wilcoxon rank sum test. A repeated measure ANOVA was performed in order to evaluate whether patients responders to PEA-um had a better outcome in the NPSI and EQ-5D scores. The analyses were performed using PASWStat 18 for Windows. Data are reported as mean values \pm standard deviations and all $P$ values are two-tailed, considering 0.05 as the statistical threshold.

\section{Results}

The main clinical and demographic characteristics of patients at baseline are reported in Table 1. During the study three subjects dropped out for reasons not related to PEA-um administration (one patient underwent surgery for colecistitis, one patient had a vertebral fracture, requiring hospitalization, and one patient had an intestinal virus); clinical data were then available for 27 patients ( 14 males and 13 females); 20/27 subjects suffered from diabetic neuropathic pain, and $7 / 27$ subjects were affected by neuropatic pain consequent to brachial plexus traumatic lesions.

At T0 evaluation VAS mean score was $8.20 \pm 1.53$ (range 6-10), NPSI total score was 5.2 \pm 1.5 (range 2. 4-8.7), and EQ$5 \mathrm{D}$ mean score was $-0.30 \pm 0.65$ (range from -1.85 to 0.65 ).
At the first evaluation (T1) the VAS mean score decreased from $8.20 \pm 1.53$ to $6.4 \pm 1.83$ (range $2-10$ ), reaching the statistical threshold $(P$ value $<0.002)$, and this improvement was even more evident at the T2 evaluation $(P$ value $<0.001)$, with a further decrease in the mean VAS score to $5.80 \pm 2.04$ (range 2-10).

Moreover, as shown in Table 2, a significant improvement was observed also in the NPSI total score, which decreased from the T0 values of $5.2 \pm 1.5$ to the T2 values of $3.8 \pm 2.1$ $(P: 0.025)$, and a similar trend was observed for the EQ-5D mean score, which increased from the T0 value of $-0.30 \pm 0.65$ to the T2 value of $0.50 \pm 0.34(P<0.001)$. According to the repeated measure ANOVA analysis, patients showing a VAS scale improvement had a significantly better outcomes at the NPSI $(P: 0.007)$ and at the EQ-5D $(P<0.001)$ scores.

A separate analysis of the NPSI different section scores (Table 2) revealed that the most evident improvement was achieved in section 5 of the scale, which refers to paresthesia and dysesthesia ( $P$ : 0.003).

\section{Discussion}

Brachial plexus neuropathy, with or without spinal cord root avulsion, and diabetic neuropathy are both frequent causes of NP, with a prevalence that may vary depending on definitions, populations, and methodologies used for the assessment of NP. Large international multicenter cohort studies $[12,13]$ report a $10-30 \%$ prevalence of NP in diabetic patients, while an Italian study described a $69 \%$ incidence of NP in 55 patients with traumatic plexopathies [14].

It has been reported that NP severity can affect the patient's quality of life more than neuropathic disability and clinical severity [15], suggesting that its symptomatic management might be one of the most important targets in the treatment of peripheral neuropathies. However, in spite of the relatively high prevalence, little consensus exists on the best primary therapeutic choices in NP, also in view of the multiple complexities underlying etiopathogenic factors.

PEA demonstrated a significant efficacy in reducing the hyperalgesic component of NP $[3,16,17]$, modulating the activity of mast cells. Moreover, PEA might help to restore the peripheral nerve sensitization process, induced by the release of inflammatory cytokines at the site of nerve injury, through a modulation of the endoneurial mast cells hyperactivity [3]. However, the highly lipophilic PEA crystalline structure has a poor oral adsorption, thus requiring to be micronized and converted into particles with an elevated surface area to volume ratio, in order to enhance its assimilation [18].

In this study, we report the first prospective short-term data of oral PEA-um treatment in patients with diabetic or traumatic NP. All subjects included in this protocol had been already treated with Pregabalin, Gabapentin, or Tramadol, with only partial improvement of symptoms and a significant residual pain, as demonstrated by the VAS score higher than 6 at the time of recruitment.

Over the 40 days observational follow-up period, we observed a significant improvement of VAS score, with an initial reduction after 10 days of therapy and a further 
TABLE 2: Baseline to follow-up scores.

\begin{tabular}{lccc}
\hline & Baseline & Followup & $P$ value \\
\hline VAS score & $8.20 \pm 1.53$ & $5.80 \pm 2.04$ & $P<0.001^{*}$ \\
NPSI total score & $5.2 \pm 1.5$ & $3.8 \pm 2.1$ & $P: 0.025^{*}$ \\
(i) Burning & $7.2 \pm 2.7$ & $5.6 \pm 3.2$ & $P: 0.063$ \\
(ii) Pressing & $5.4 \pm 2.9$ & $4.0 \pm 3.0$ & $P: 0.073$ \\
(iii) Paroxysmal pain & $5.6 \pm 3.1$ & $4.0 \pm 3.0$ & $P: 0.061$ \\
(iv) Evoked pain & $3.7 \pm 2.3$ & $3.0 \pm 2.2$ & $P: 0.279$ \\
(v) Paresthesia/dysesthesia & $5.9 \pm 2.6$ & $3.6 \pm 2.9$ & $P: 0.003^{*}$ \\
EQ-5D score & $-0.30 \pm 0.65$ & $0.50 \pm 0.34$ & $P<0.001^{*}$ \\
\hline
\end{tabular}

A significant improvement was observed in the VAS and NPSI scores between baseline and followup. Moreover, the NPSI subscores showed a highly significant improvement for the paresthesia/dysesthesia subscore and a trend towards amelioration for the other subscores.

VAS: Visual Analogue Scale; NPSI: Neuropathic Pain Symptom Inventory; EQ-5D: health questionnaire five dimensions.

${ }^{*}$ Indicates the statistical significance $(P$ value $<0.05)$.

decrease at the end of the study. In addition, also the NPSI score related to paresthesia and dysesthesia significantly improved, as well as the quality of life measured with the EQ$5 \mathrm{D}$ scale.

Overall, our findings would suggest that PEA-um oral administration might be effective as an add-on therapy in the control of NP, also resulting in an amelioration of patient quality of life. However, two limitations should be considered in the interpretations of our data: this study reports the short-term follow-up data of a single center open-label study, and the absence of a comparative group does not allow speculations on the possible placebo effect in relieving pain. Further larger prospective studies with longer follow-up duration seem therefore necessary to confirm our findings and the long-term possible efficacy of oral PEA-um treatment in the management of peripheral neuropathic pain.

\section{Conflict of Interests}

The authors declare that there is no conflict of interests regarding the publication of this paper.

\section{Acknowledgments}

This research was funded by the "Associazione Neuropatie Croniche Piemonte ONLUS." The sponsor had no role either in the design of the study or in the collection, analysis, and interpretation of the data or in the decision to approve publication of the finished paper.

\section{References}

[1] H. MacHelska, "Dual peripheral actions of immune cells in neuropathic pain," Archivum Immunologiae et Therapiae Experimentalis, vol. 59, no. 1, pp. 11-24, 2011.

[2] R. S. Duncan, K. D. Chapman, and P. Koulen, "The neuroprotective properties of palmitoylethanolamine against oxidative stress in a neuronal cell line," Molecular Neurodegeneration, vol. 4, no. 1, article 50, 2009.
[3] B. Costa, F. Comelli, I. Bettoni, M. Colleoni, and G. Giagnoni, "The endogenous fatty acid amide, palmitoylethanolamide, has anti-allodynic and anti-hyperalgesic effects in a murine model of neuropathic pain: involvement of CB1, TRPV1 and PPAR $\gamma$ receptors and neurotrophic factors," Pain, vol. 139, no. 3, pp. 541550, 2008.

[4] R. S. Calabrò, G. Gervasi, S. Marino, P. N. Mondo, and P. Bramanti, "Misdiagnosed chronic pelvic pain: pudendal neuralgia responding to a novel use of palmitoylethanolamide," Pain Medicine, vol. 11, no. 5, pp. 781-784, 2010.

[5] U. Indraccolo and F. Barbieri, "Effect of palmitoylethanolamidepolydatin combination on chronic pelvic pain associated with endometriosis: preliminary observations," European Journal of Obstetrics Gynecology and Reproductive Biology, vol. 150, no. 1, pp. 76-79, 2010.

[6] L. Cobellis, M. A. Castaldi, V. Giordano et al., "Effectiveness of the association micronized N-Palmitoylethanolamine (PEA)transpolydatin in the treatment of chronic pelvic pain related to endometriosis after laparoscopic assessment: a pilot study," European Journal of Obstetrics Gynecology and Reproductive Biology, vol. 158, no. 1, pp. 82-86, 2011.

[7] R. Conigliaro, V. Drago, P. S. Foster, C. Schievano, and V. Di Marzo, "Use of palmitoylethanolamide in the entrapment neuropathy of the median in the wrist," Minerva Medica, vol. 102, no. 2, pp. 141-147, 2011.

[8] A. Truini, A. Biasiotta, G. Di Stefano et al., "Palmitoylethanolamide restores myelinated-fibre function in patients with chemotherapy-induced painful neuropathy," CNS and Neurological Disorders-Drug Targets, vol. 10, no. 8, pp. 916-920, 2011.

[9] K. J. Hunt and D. Hurwit, "Use of patient-reported outcome measures in foot and ankle research," The Journal of Bone and Joint Surgery, vol. 95, article 118, 2013.

[10] L. Padua, C. Briani, S. Jann et al., "Validation of the italian version of the neuropathic pain symptom inventory in peripheral nervous system diseases," Neurological Sciences, vol. 30, no. 2, pp. 99-106, 2009.

[11] R. Rabin, C. Gudex, C. Selai, and M. Herdman, "From translation to version management: a history and review of methods for the cultural adaptation of the euroQol five-dimensional questionnaire," Value Health, vol. 17, pp. 70-76, 2014.

[12] C. A. Abbott, R. A. Malik, E. R. van Ross, J. Kulkarni, and A. J. Boulton, "Prevalence and characteristics of painful diabetic neuropathy in a large community-based diabetic population in the U.K," Diabetes Care, vol. 34, pp. 2220-2224, 2011.

[13] P. J. Dyck, K. M. Kratz, J. L. Karnes et al., "The prevalence by staged severity of various types of diabetic neuropathy, retinopathy, and nephropathy in a population-based cohort: the Rochester Diabetic Neuropathy Study," Neurology I, vol. 43, no. 4, pp. 817-824, 1993.

[14] P. Ciaramitaro, M. Mondelli, F. Logullo et al., “Traumatic peripheral nerve injuries: epidemiological findings, neuropathic pain and quality of life in 158 patients," Journal of the Peripheral Nervous System, vol. 15, no. 2, pp. 120-127, 2010.

[15] D. Cocito, I. Paolasso, C. Pazzaglia et al., "Pain affects the quality of life of neuropathic patients," Neurological Sciences, vol. 27, no. 3, pp. 155-160, 2006.

[16] L. Facci, R. Dal Toso, S. Romanello, A. Buriani, S. D. Skaper, and A. Leon, "Mast cells express a peripheral cannabinoid receptor with differential sensitivity to anandamide and palmitoylethanolamide," Proceedings of the National Academy of Sciences of the United States of America, vol. 92, no. 8, pp. 33763380, 1995. 
[17] W. P. Farquhar-Smith, S. I. Jaggar, and A. S. C. Rice, "Attenuation of nerve growth factor-induced visceral hyperalgesia via cannabinoid CB1 and CB2-like receptors," Pain, vol. 97, no. 1-2, pp. 11-21, 2002.

[18] S. Rao, Y. Song, F. Peddie, and A. M. Evans, "Particle size reduction to the nanometer range: a promising approach to improve buccal absorption of poorly water-soluble drugs," International Journal of Nanomedicine, vol. 6, pp. 1245-1251, 2011. 


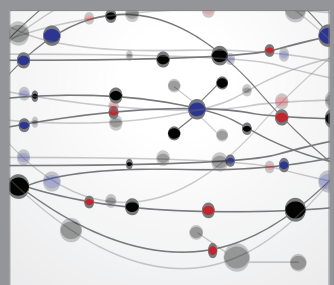

The Scientific World Journal
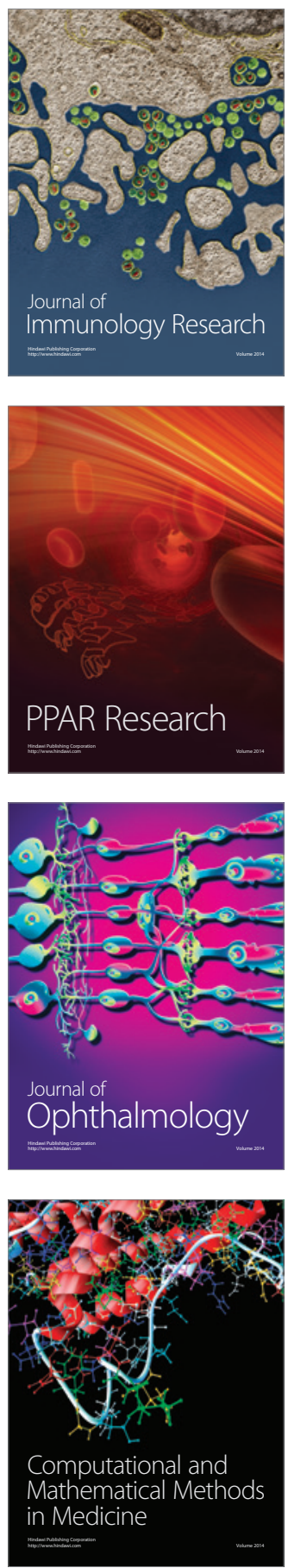

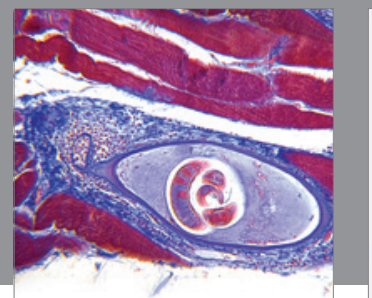

Gastroenterology

Research and Practice
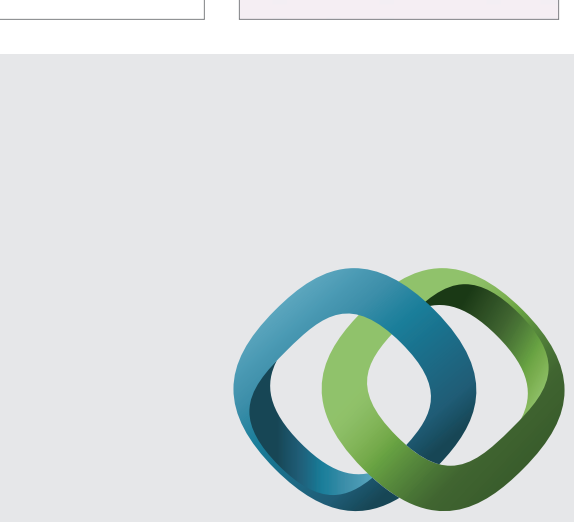

\section{Hindawi}

Submit your manuscripts at

http://www.hindawi.com
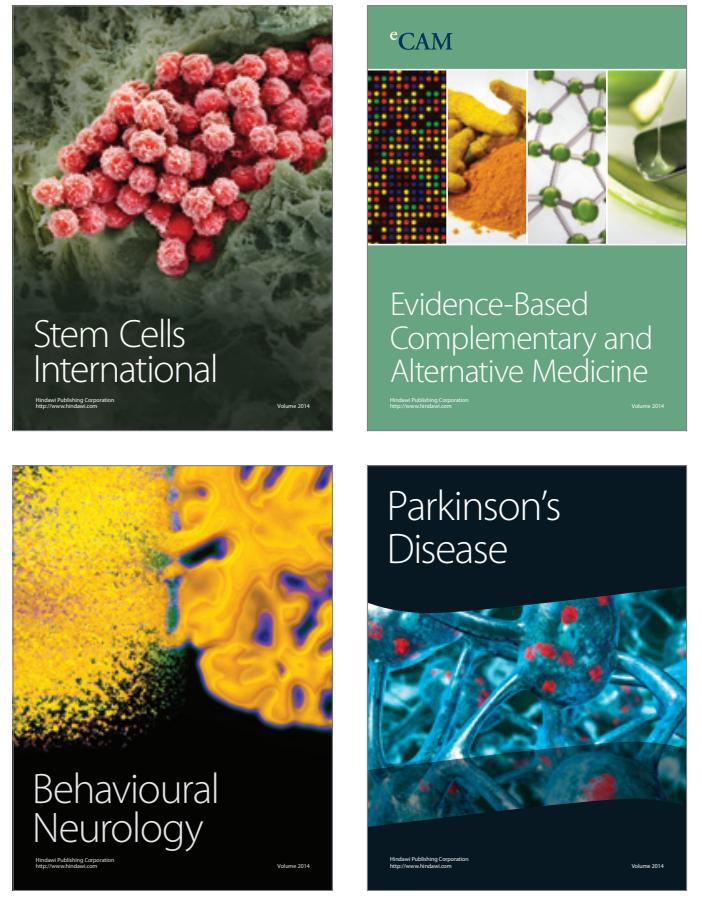
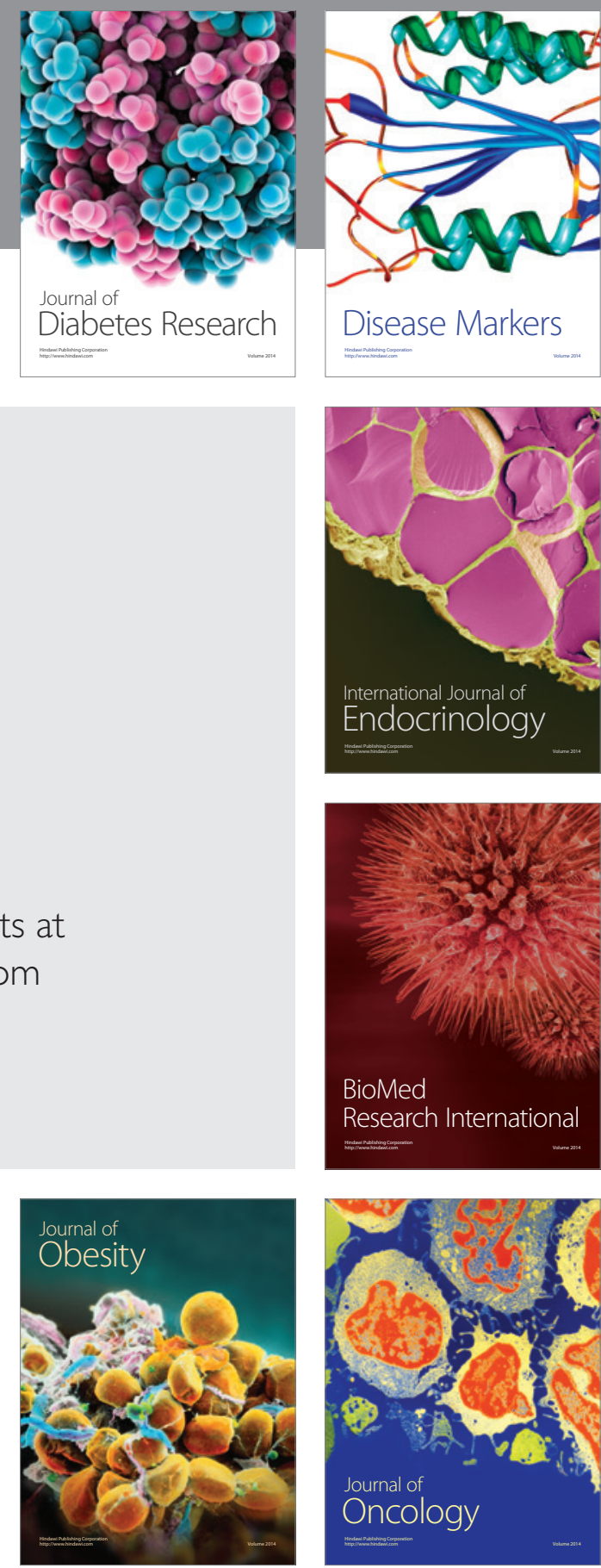

Disease Markers
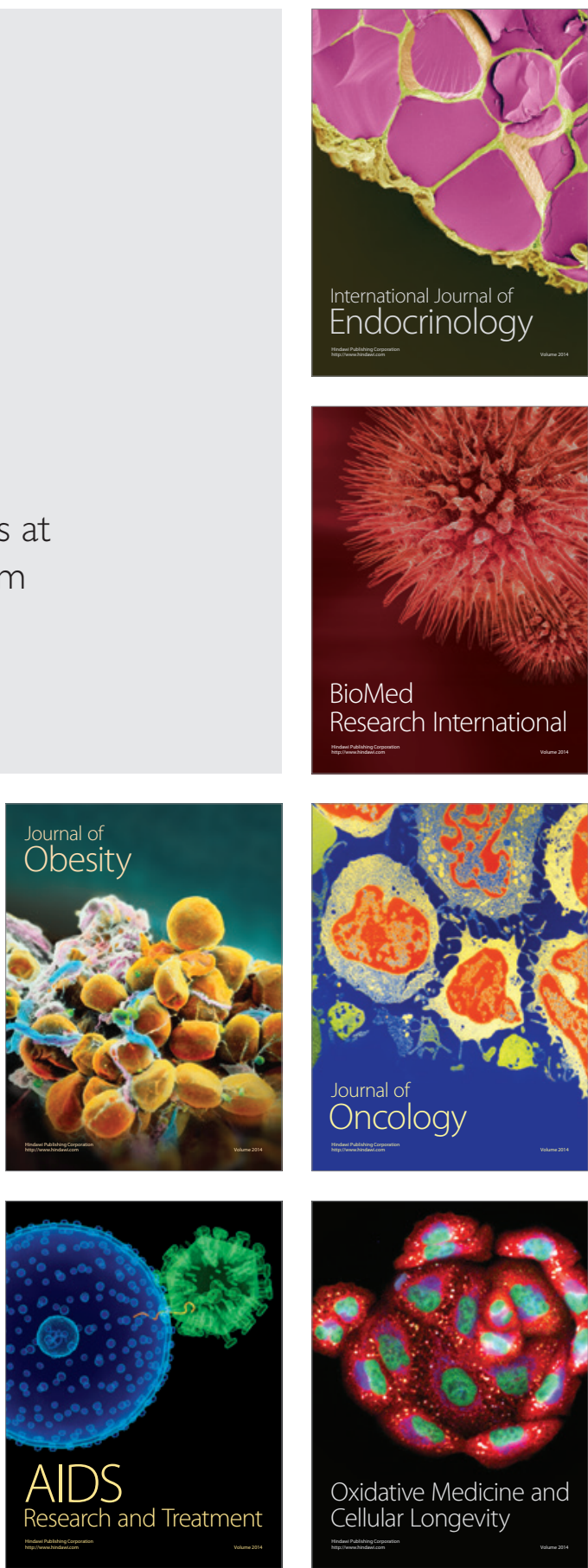